\title{
Effect of Pre-processing on Satellite Image Fusion
}

\author{
Yoonsuk Choi*, Ershad Sharifahmadian, Shahram Latifi \\ Dept. of Electrical and Computer Engineering, University of Nevada, Las Vegas \\ 4505 Maryland Parkway, Las Vegas, NV 89154-4026
}

\begin{abstract}
Satellite image fusion plays a key role in providing researchers with more precise and abundant information by injecting spatial details of a panchromatic image into a multispectral image. However, pre-processing of source images is required prior to the fusion process. Without well-performed pre-processing, the main fusion process cannot produce highquality fusion results because incorrect registration of the source images can significantly degrade the fusion results either spatially or spectrally. Therefore, we analyze the effect of preprocessing on satellite image fusion in this study, using four differently pre-processed data sets. In addition, fusion framework and fusion scheme are based on the contourlet transform which outperforms the wavelet transform in terms of representing salient features of the source images. As a result of both spatial and spectral analyses, we conclude that coregistration and pre-processing of the source images can significantly improve the quality of the fusion results.
\end{abstract}

Keywords—contourlet transform; multispectral; panchromatic; registration; wavelet

\section{INTRODUCTION}

Multispectral image has higher spectral resolution while panchromatic image has higher spatial resolution. Therefore, image fusion is applied to produce an image which provides high resolution both spatially and spectrally. Image fusion can fuse more than one input image to produce an enhanced result by integrating all the positive advantages of the input images because each input image can be acquired from a different source with unique characteristics. Among various image fusion techniques, satellite image fusion for multispectral (MS) and panchromatic (PAN) images has gained remarkable attention over the past few years as it can provide researchers with both spatial and spectral resolution significantly improved.

Many efforts have been made to fuse both multispectral and panchromatic images. The standard image fusion techniques based on IHS, PCA, and Brovey transforms usually produce poor results; hence, new fusion schemes like wavelet transform have been proposed to address particular problems of the standard techniques. Wavelet transform which is essentially extension of the idea of high-pass filtering has been recognized as having great potential in image processing applications [1]. During the fusion process, the extracted detail information from one image can be injected into another image using different methods; for example, substitution, addition and selection method based on either frequency or spatial context. Furthermore, the wavelet function used in the transform can be designed to have specific properties that are useful in the particular application of the transform [2]. Although the wavelet-based fusion schemes produce positive results, they also have drawbacks that must be overcome in the process of image fusion. The wavelet transform is good at isolating the discontinuities at object edges, but this transform cannot detect the smoothness along the image edges. Moreover, it can only capture limited directional information. In 2005, a new idea of contourlet transform was proposed by Do and Vetterli [3]. The contourlet transform is a new framework of discrete image with multi-scalability and multi-directionality, which can capture intrinsic geometric structure information of images and achieve better expression than discrete wavelet transform. Recent contourlet based fusion methods can be found in the literature [4]-[6].

Since the contourlet transform can effectively overcome the disadvantages of the wavelet transform, we employ the contourlet-based fusion framework and scheme in our study. In addition to the main fusion process, pre-processing of the source images is another important process that we need to go through in order to produce high-quality fusion results. Although the fusion framework and scheme are very advanced, it is not possible to obtain high-quality fusion results without pre-processing the source images correctly. Therefore, it is necessary to examine how pre-processing of the source images affects the fusion results.

In this paper, we first briefly explain the contourlet transform in Section II. In Section III, we discuss the fusion framework, fusion scheme and performance quality metrics. Next, we discuss the pre-processing methods in Section IV, and experimental study and analysis in Section V. Lastly, we conclude in Section VI.

\section{CONTOURLET TRANSFORM}

The wavelet transform is good at isolating the discontinuities at object edges, but cannot detect the smoothness along the edges. Moreover, the wavelet transform can only capture limited directional information. The contourlet transform is a multi-scale and multi-direction framework of discrete image, and can effectively overcome the disadvantages of the wavelet transform. In this transform, multi-scale analysis and multi-direction analysis are separated in serial. The Laplacian pyramid (LP) [7] is first used to capture the point discontinuities, then followed by a directional filter bank (DFB) [8] to link point discontinuities to linear structures. The overall result is an image expansion using basic elements like contour segments. The contourlet transform framework is depicted in Fig. 1. 


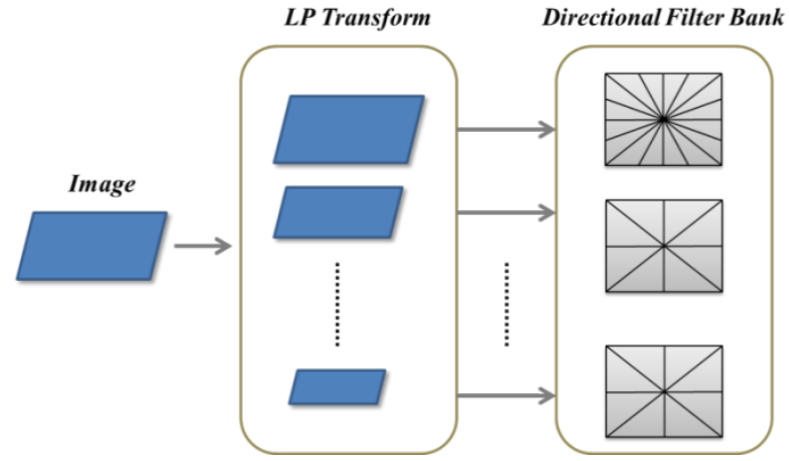

Fig. 1. The contourlet transform framework.

Fig. 2 shows the contourlet filter bank. First, multi-scale decomposition is processed by the Laplacian pyramid, and then the directional filter bank is applied to each band pass channel.

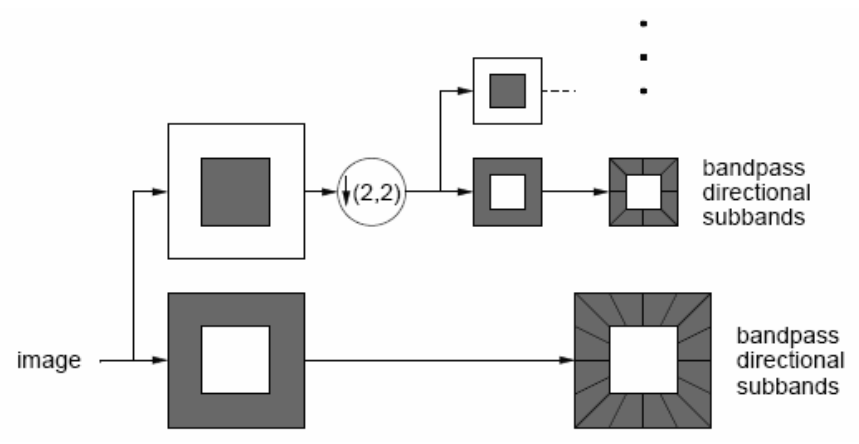

Fig. 2. The contourlet filterbank.

The contourlet expansion of images consists of basis images oriented at various directions in multiple scales with flexible aspect ratio. In addition to retaining the multi-scale and time-frequency localization properties of wavelets, the contourlet transform offer high degree of directionality. Contourlet transform also adopts nonseparable basis functions, which capture the geometrical smoothness of the contour along any possible directions. Compared with traditional image expansions, contourlet can capture 2-D geometrical structure in natural images much more efficiently [9].

Furthermore, in image enhancement, wavelet-based methods present some limitations because they are not well adapted to the detection of highly anisotropic elements such as alignments in an image. Contourlet transform has better performance in representing the salient image features, such as edges, lines, curves and contours due to its anisotropy and directionality.

\section{IMAGE FUSION}

\section{A. Fusion Framework}

The fusion framework used in the experiments is shown in Fig. 3. First, source images are decomposed into multi-scale and multi-directional components using contourlet transform, and these components are fused together based on a certain fusion scheme. Next, inverse contourlet transform is performed in order to obtain a final fused image.

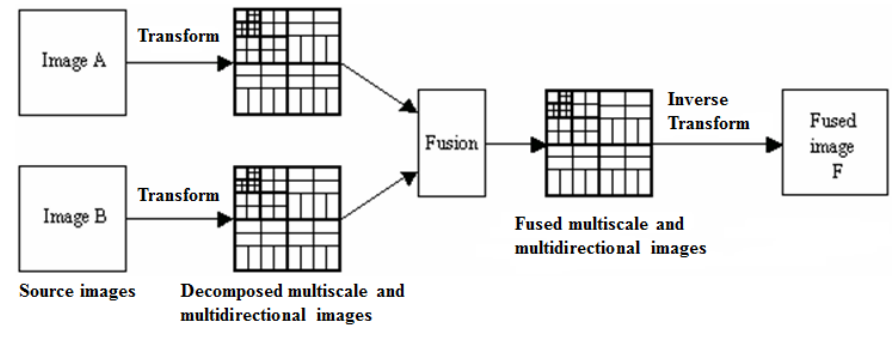

Fig. 3. Fusion framework.

\section{B. Fusion Scheme}

All four differently pre-processed data sets are fused according to the fusion scheme and fusion rule that are described as follows:

1) The source images are decomposed using contourlet transform in order to obtain multi-scale or multi-directional frequency coefficients. In our experiments, decomposition level of 3 was used since the level beyond 3 did not give significant improvement.

2) The maximum frequency fusion rule is used for the fusion of the frequency coefficients. In other words, higher frequency coefficients are selected from each set, and these selected frequency coefficients are used as coefficients of the fused image.

3) The inverse transform step is performed to obtain the final fusion image. The fused coefficients are subjected to the inverse transform.

\section{Performance Quality Metrics}

Fusion results are compared using various quality assessment parameters. We used correlation coefficient (CC), relative average spectral error (RASE) and spectral angle mapper (SAM) for spectral analysis. To spatially analyze the fusion results, we employed distortion extent (DE), universal image quality index (UIQI) and signal to noise ratio (SNR).

\section{PRE-PROCESSING MethodS}

When satellite image fusion is performed, it is very important to pre-process the source images correctly. However, in most cases, it is not easy to find two perfectly co-registered images for the fusion process. Therefore, the source images should be carefully pre-processed to make them co-registered to each other as precisely as possible. In our study, we preprocess the original source images (introduced in Section V) in four different ways in order to analyze how each method affects the fusion results. The four different pre-processing methods are as follows:

1) MS image is resampled to match the scale of PAN image. Two source images (PAN and MS) are assumed to be co-registered to each other. As a result, we use the resampled MS image and the original PAN image as two source images, and the original MS image as a reference image. 
2) MS image is resampled to match the scale of PAN image. Then, two source images (PAN and MS) are coregistered using intensity-based automatic image registration. As a result, we use a pair of co-registered images (resampled MS image and original PAN image) as two source images, and the original MS image as a reference image.

3) Instead of using two original source images, only original MS image is used to obtain two synthesized source images. First, the original MS image is down sampled by a factor of 3 and resized to the same size as before using the bilinear interpolation. Second, the band 1 of the original MS image is selected to create a panchromatic image with comparatively high spatial resolution. As a result, we obtain two perfectly co-registered images with a threefold difference in spatial resolution. These synthesized images can be used as a pair of source images and the original MS image can be used as a reference which the fusion results are compared to.

4) Similar to the third method, the original MS image is synthesized to make a pair of source images. First, the original MS image is down sampled by a factor of 3 and resized to the same size as before using the bilinear interpolation. In order to get another source image, PAN image, the original MS image is spectrally integrated over the entire spectral range. The final result is a synthesized PAN image that can be used as the second source image [10]. By doing this, we can obtain two perfectly co-registered source images without going through the registration process which takes up lots of time and computational load.

\section{EXPERIMENTAL STUDY AND ANALYSIS}

In this study, we employ a pair of MS and PAN images [11] to analyze effects of pre-processing on satellite image fusion. The MS and PAN images were acquired by QuickBird satellite over the area of Egypt with resolution of $0.7 \mathrm{~m}$ and $2.8 \mathrm{~m}$ respectively. Pre-processing was first conducted over the original source images to produce four differently preprocessed data sets as discussed in Section IV. After the preprocessing, we performed the image fusion on four different data sets using the contourlet-based fusion framework and fusion scheme as explained in Section II and III. We can see the original PAN and MS images in Fig. 4. In Fig. 5, two pairs of source images obtained using pre-processing methods 1 and 2 are shown respectively. Note that the only difference between method 1 and 2 is the co-registration. In Fig. 6, two pairs of source images obtained using method 3 and 4 are shown respectively. Lastly, the fusion results of four different pre-processing methods are shown in Fig. 7.

As shown in Table 1, the fusion results vary by preprocessing methods. In method 1 , the original PAN image and the resampled MS image are fused together, and the fusion result is compared to the reference MS image. However, the source images were not co-registered to each other. The second method is identical to the first method except the fact that the source images are co-registered to each other. On the other hand, the third and fourth methods use the perfectly coregistered source images by synthesizing the original MS image. Based on our analysis, methods 3 and 4 outperform methods 1 and 2, and provide high resolution both spectrally and spatially. Better performance means that the fusion results have higher resolution with more abundant information. In addition, although both methods 3 and 4 use perfectly coregistered source images, we can see notable differences in between. The third method performs better spatially because the band 1 was selected directly from the original MS image to be used as a PAN source image. By doing this, the spatial data were not distorted. In contrast, the fourth method performs better spectrally because a PAN source image was obtained by spectrally integrating the original MS image over the entire spectral range. As a result, the spectral data were preserved with as less distortion as possible.

\section{CONCLUSION}

Image fusion is undoubtedly important area of study since it provides users with more precise and abundant information. In particular, satellite image fusion takes a huge role in producing images with both high-spatial and high-spectral resolutions. In our study, we created four different data sets by pre-processing the original pair of PAN and MS images in four different ways. For all of the data sets, we applied the same fusion framework and fusion scheme based on the contourlet transform. The fusion results were quantitatively analyzed both spatially and spectrally, using various quality metrics. As a result, the last two data sets which were obtained from preprocessing methods 3 and 4 outperformed the first two data sets which were obtained from pre-processing methods 1 and 2 . The result was true for both spatial and spectral analyses. However, notable differences were examined between the last two data sets. In other words, the third data set outperformed the fourth data set in terms of spatial analysis, and the fourth data set outperformed the third data set in terms of spectral analysis. The difference occurred due to the pre-processing method of obtaining panchromatic source images, as discussed in Section V. Our future research will be focused on finding a novel pre-processing method which can improve the satellite image fusion results in both spatial and spectral resolutions.

\section{ACKNOWLEDGMENT}

This work was supported (in part) by the Defense Threat Reduction Agency, Basic Research Award \# HDTRA1-12-10033, and the Nevada EPSCoR Programs, NSF EPSCoR Track I, 2013-2018.

\section{REFERENCES}

[1] Graps, A., "An introduction to wavelets. IEEE Computational Science and Engineering”, 50-61, 1995.

[2] Mallat, S.G., "A Wavelet Tour of Signal Processing", second ed. Academic Press, San Diego, 1999.

[3] M. N. Do and M. Vetterli, "The Contourlet Transform: An Efficient Directional Multiresolution Image Representation", IEEE Transactions On Image Processing, vol. 14, no. 12, pp. 2091-2106, Dec. 2005.

[4] Sun, Xiangda; Du, Junping; Li, Qingping; Li, Xiaoyong; Xu, Liang; Li, Yawen, "Improved energy contrast image fusion based on nonsubsampled contourlet transform," Industrial Electronics and Applications (ICIEA), 2013 8th IEEE Conference on , vol., no., pp.1610,1613, 19-21 June 2013.

[5] Yazdi, M.; Ghasrodashti, E.K., "Image fusion based on NonSubsampled Contourlet Transform and phase congruency," Systems, 
Signals and Image Processing (IWSSIP), 2012 19th International Conference on , vol., no., pp.616,620, 11-13 April 2012.

[6] Xiaorong Xue; Aimin Wang; Hongfu Wang; Fang Xiang, "A fusion method of multi-spectral image and panchromatic image based on NSCT and IHS transform," Intelligent Control and Information Processing (ICICIP), 2012 Third International Conference on , vol., no., pp.640,643, 15-17 July 2012.

[7] Burt P J., "Merging images through pattern decomposition", Proceedings of SPIE, 575: 173-18, 1985.

[8] Bamberger R H., "A filter bank for the directional decomposition of images: Theory and design", IEEE Trans. Signal Processing, 40 (4): 882 $-893,1992$.
[9] Aboubaker M. ALEjaily et al., "Fusion of remote sensing images using contourlet transform", Innovations and Advanced Techniques in Systems, Computing Sciences and Software Engineering, Springer, pp. 213-218, 2008

[10] M. Eismann, R. Hardie, "Application of the stochastic mixing model to hyperspectral resolution enhancement", IEEE Transactions on Geoscience and Remote Sensing 42 (9) (2004) 1924-1933.

[11] http://studio.gge.unb.ca/UNB/zoomview/examples.html

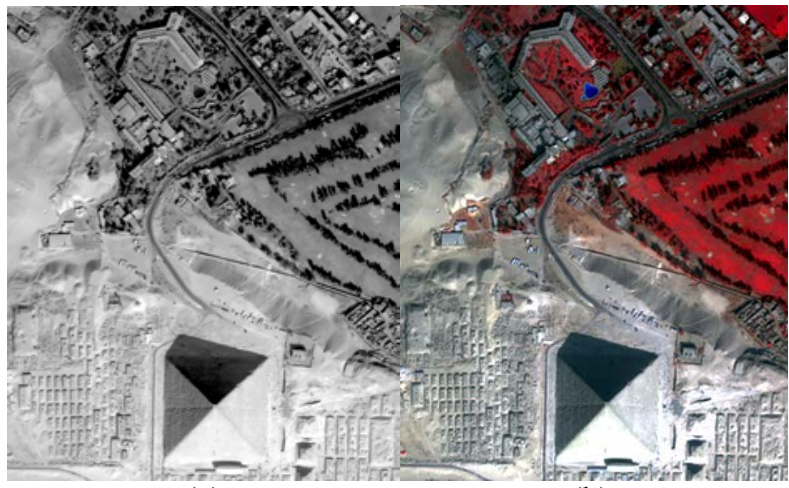

(a) (b)

Fig. 4. Two original source images. (a) Original PAN image. (b) Original MS image..

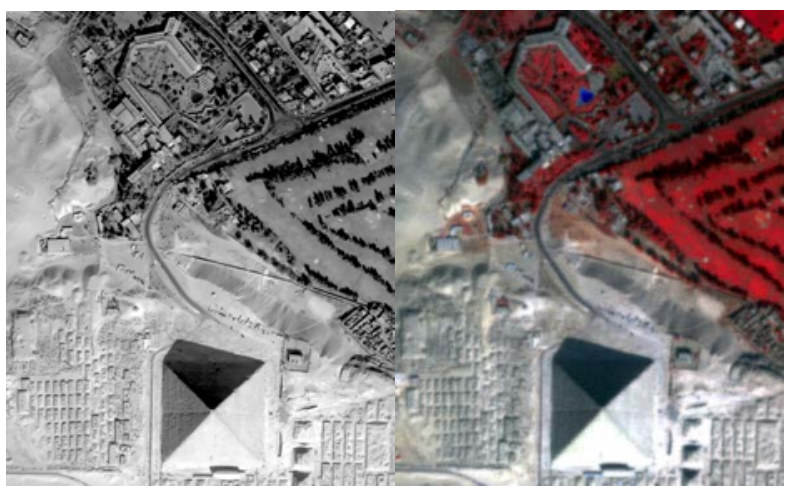

(a)

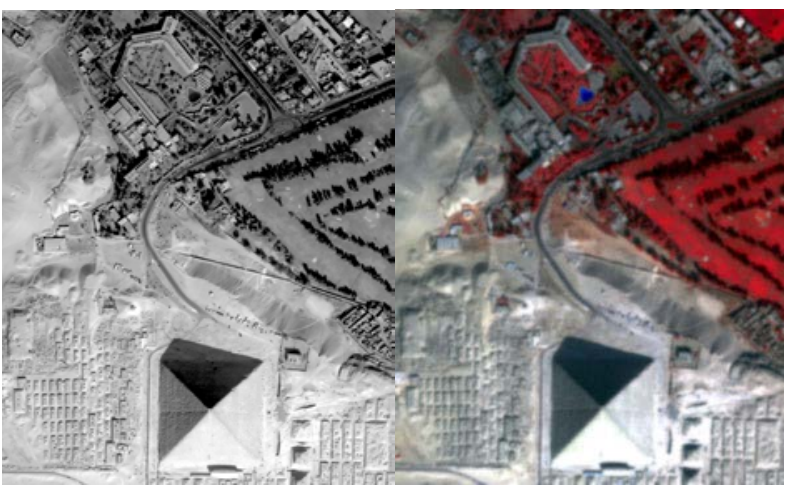

(b)

Fig. 5. Two pairs of source images obtained from pre-processing methods 1 and 2. (a) Method 1. (b) Method 2.

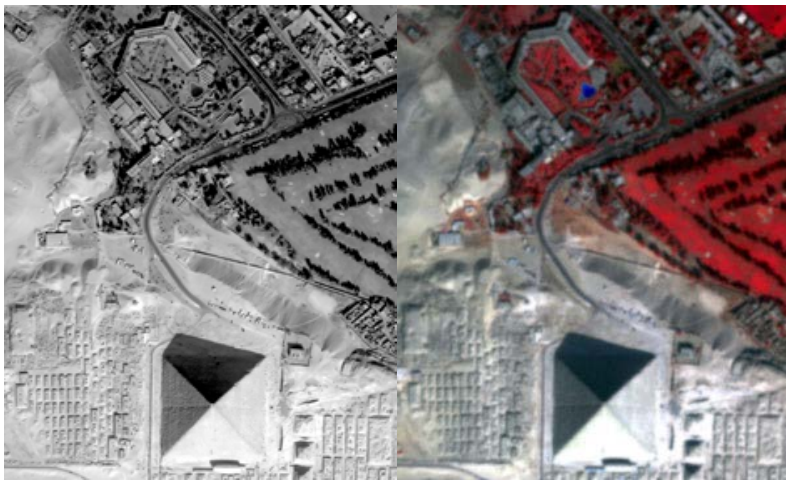

(a)

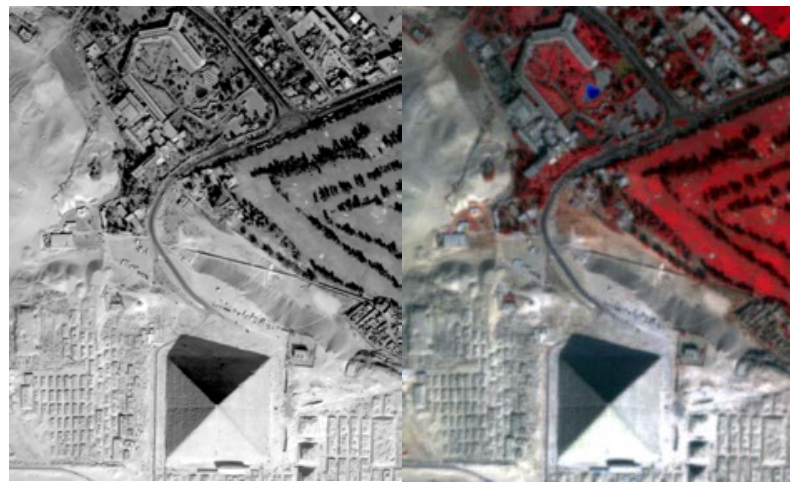

(b)

Fig. 6. Two pairs of source images obtained from pre-processing methods 3 and 4. (a) Method 3. (b) Method 4. 


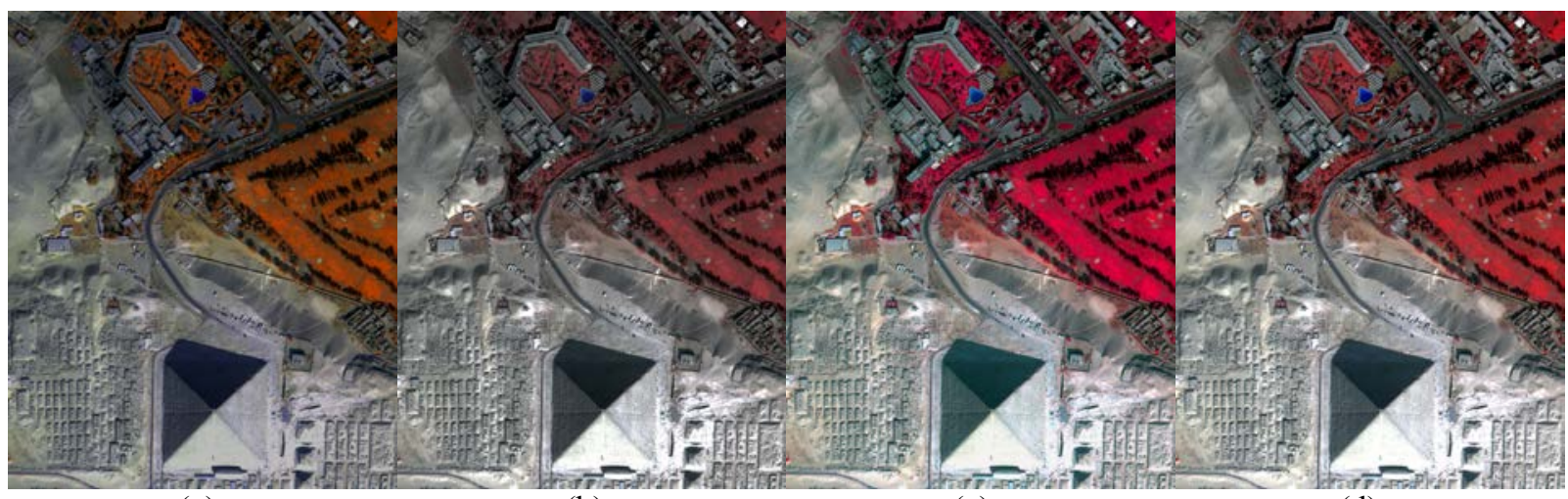

(a)

(b)

(c)

(d)

Fig. 7. Fusion results of four differently pre-processed data sets. (a) Method 1. (b) Method 2. (c) Method 3. (d) Method 4.

TABLE I. A COMParison of Fusion Results using Performance Quality Metrics

\begin{tabular}{|c|c|c|c|c|c|c|}
\hline \multirow{2}{*}{$\begin{array}{l}\text { Pre-processing } \\
\text { Method }\end{array}$} & \multicolumn{3}{|c|}{ Spectral Analysis } & \multicolumn{3}{|c|}{ Spatial Analysis } \\
\hline & $C C$ & $R A S E$ & $S A M$ & $D E$ & UIQI & SNR \\
\hline Method 1 & 0.857 & 44.813 & 0.286 & 29.246 & 0.586 & 68.875 \\
\hline Method 2 & 0.873 & 44.772 & 0.275 & 29.081 & 0.624 & 69.137 \\
\hline Method 3 & 0.885 & 44.582 & 0.262 & 28.693 & 0.731 & 69.859 \\
\hline Method 4 & 0.892 & 44.578 & 0.258 & 28.742 & 0.726 & 69.823 \\
\hline
\end{tabular}

